\title{
Evolution of Viscoelasticity through Sol-Gel Transition of Polyacrylonitrile/Dimethylacetamide Solution : Power Law Relaxation observable with Characteristic Frequency Window
}

\author{
Y. Tanaka, M. Arimoto \\ Department of Materials Science and Engineering, Faculty of Engineering, University of Fukui, Fukui 910-8507, Japan \\ Fax: 81-(0)776-27-8767, e-mail: tanaka@matse.u-fukui.ac.jp
}

\begin{abstract}
Thermally induced gelation of polyacrylonitrile(PAN) solution in dimethylacetamide was studied through dynamic viscoelasticity measurements. PAN gel were prepared by the freezing $\left(-50^{\circ} \mathrm{C}\right)$ and thawing $\left(25^{\circ} \mathrm{C}\right)$ method. The evolution of $G^{\prime}(\omega)$ and $G^{\prime \prime}(\omega)$ data in the process of gelation is observed and the critical behaviour is obtained around the sol-gel threshold in which the power law of $G^{\prime}(\omega) \sim G^{\prime \prime}(\omega) \propto \omega^{n}$ holds. The evolution in the gelation can be interpreted through the dynamic viscosity, in which characteristic frequency window is defined in connection with the formation of PAN network. The relation between the frequency and the correlation length is also found. The correlation length characterise the size of the cluster formed by the aggregation of PAN chain in pre-gel state, and also characterise the size of regions within the network to follow the power law.

Key words : $\quad$ Polyacrylonitrile, Gelation, Freezing and Thawing, Viscoelasticity, Power Law Relaxation
\end{abstract}

\section{INTRODUCTION}

In the previous work, viscoelastic behaviour for the solution of polyacrylonitrile - N,Ndimethylacetamide was investigated concerning sol-gel transition [1]. The gelation was induced by the freezing and thawing method [2], where the gel elasticity and the freezing time were examined. In addition, the power law relaxation was observed at the gel point, from which the index of gelation was deduced. In evaluating the indices, it was found that the power law relaxation observed could also be discussed in terms of the relationship between the network structure and the frequency window used in the rheology measurement.

The relationship is derived from the concept of mechanical self-similarity, which was proposed by Vilgis and Winter, and applied to the experimental results of the end-linking network formation $[3,4]$. Although the concept is very interesting from the viewpoint of the relation between $G^{\prime}, G^{\prime \prime}$ data and the geometry of network, no other research reports seem to follow this analysis. In this paper, discussion on polymer network of polyacrylonitrile (PAN) will be given on the basis of the analyses of the frequency window, which is of significance in the sense of geometrical structure of network in physical gel.

It is important, in general, to clarify the composition of the junction zone as examining the network structure. As for the crystallinity in the gelation process, some experimental studies can be seen for PAN gels, although the complete prove that crystallinity is the basis for the gelation has not yet been shown. According to Beckmann and Zenke[5], the junction zone might be composed of stable nuclei, where further growth is hindered in the chain direction due to stereo-irregularity of PAN molecules, or the result of nucleation processes due to phase separation in the binodal region of liquid-liquid demixing. It is also suggested that the composition of junction is influenced by the type of sample(reactor powder, oriented fibres, single crystals grown from dilute solutions, etc). Accordingly, the junction zone in PAN gels is somewhat a controversial issue.

The sample in this work is prepared with suspension polymerisation in water system, more precisely, it is called as dispersion polymerisation since the powdered polymer is simultaneously separated from the aqueous monomer solution. In reactor powder, it is reported to be rather easy to observe crystallinity [6]. And therefore, it may be said that the crosslinking loci, it is equivalent to the junction, are composed of some ordered structure like crystals.

The discussion section of this paper is concerned with the branched macromolecule cluster formed by the crosslinking. It has flexible chains of arbitrary self-similar connectivity, and thus the fractal geometry is applied. In addition, the branch units have different functionalities, that is, the active sites are distributed on the branched unit to form crosslinking loci. The presence of loops can also be adequately described in the branched macromolecule cluster. Furthermore, detailed relations between the power law, fractal geometry, self-similar structure are suggested in many papers in terms of the dynamics of branched macromolecular cluster and polymer network $[3,4,7,8]$. With these backgrounds, the analyses of network structure is developed on the basis of $G^{\prime}, G^{\prime \prime}$ data of PAN gels following the research works of Vilgis and Winter. 
The data already appeared in the previous paper were used in part again for reference[1].

\section{EXPERIMENTAL}

Polyacrylonitrile was kindly supplied from Mitsubishi Rayon Co., Ltd., and used without further purification. The viscosity average molecular weight was determined from the following equation [9], to be $\bar{M}=1.3 \times 10^{5}$.

$$
[\eta]=3.35 \times 10^{-4} \times \bar{M}^{0.72}
$$

GPC measurement was carried out at $50^{\circ} \mathrm{C}$, which determined $M_{\mathrm{w}} / M_{\mathrm{n}}$ to be 2.4. $N, N$-Dimethylacetamide (DMA) was purchased from Nacalai. inc. and dehydrated. DMA has a freezing point of $-20^{\circ} \mathrm{C}[10]$. After preparing the solution it was soaked to a bath of $-50^{\circ} \mathrm{C}$ for various hours, then poured into a measurement cell of the viscoelasticity analyser (ARES, Rheometric Scientific F.E.) which was kept to be $25^{\circ} \mathrm{C}$. In $-50^{\circ} \mathrm{C}$, the solution is completely turbid, i.e., it is absolutely frozen. Accordingly, the freezing temperature in the experiment is $-50^{\circ} \mathrm{C}$, the temperature of thawing is $25^{\circ} \mathrm{C}$. In the text that follows, the length while a solution was frozen will be referred to as the freezing time $\left(t_{\mathrm{F}}\right)$, the length that solution was kept in the cell of $25^{\circ} \mathrm{C}$ will be referred to as the aging time $\left(t_{\mathrm{A}}\right)$. The range of $t_{\mathrm{F}}$ is from 2 to $17.5 \mathrm{~h}$. The polymer concentration is fixed to $14 \%$ by weight. See the previous paper for other experimental description [1].

\section{RESULTS AND DISCUSSION}

3.1 Evolution of Viscoelasticity through Sol-Gel Transition

Figure 1 shows the $G^{\prime}(\omega)$ and $G^{\prime \prime}(\omega)$ data over the double logarithmic plot as a function of $t_{\mathrm{A}}$ for the solution of $t_{\mathrm{F}}=17.5 \mathrm{~h}$. Before the solution was frozen, the measurement was also carried out to find the liquid like behaviour of $G^{\prime}(\omega) \propto \omega^{2}$ and $G^{\prime \prime}(\omega) \propto \omega \quad(\omega \rightarrow 0)$, which was approximately observed for the data of $t_{\mathrm{A}}=30 \mathrm{~min}$ in Figure 1 . $G^{\prime}(\omega)$ and $G^{\prime \prime}(\omega)$ data deviate from these relations with the increase in $t_{\mathrm{A}}$, which reflects the evolution of network formation. And eventually, plateau region appears in $G^{\prime}(\omega)$ data of $t_{\mathrm{A}}=$ $2054 \mathrm{~min}$ indicating the formation of gel elasticity; the terminal region in $G^{\prime}(\omega)$ still shows the tendency of falling which will be discussed later. The slope of the $G^{\prime}(\omega)$ vs $\omega$ curve $($ at $\omega \rightarrow 0)$ on the double logarithmic scale tends to change from 2 to 0 (the gel elasticity), while that of $G^{\prime \prime}(\omega)$ vs $\omega$ curve (at $\omega \rightarrow 0$ ) varies from 1 to 0 . Figure 1 shows that the liquid-solid transition takes place while keeping the solution at $25^{\circ} \mathrm{C}$.

It was also confirmed by extracting $G^{\prime}(\omega)$ and $G^{\prime \prime}(\omega)$ data of $t_{\mathrm{A}}=998 \mathrm{~min}$ that the power law relaxation of $G^{\prime}(\omega)$ and $G^{\prime \prime}(\omega) \propto \omega^{n}$ is observed over three decades of $10^{-2} \leq \omega \leq 10 \mathrm{rad} / \mathrm{s}$ where $G^{\prime}$ and $G^{\prime \prime}$ plots are apparently parallel to each other. It can be thought of as the gel point; this ageing time is referred to as $t_{\mathrm{A} \text {-gel }}$. $G^{\prime \prime}$ values were higher than $G^{\prime}$ over all frequency range of $10^{-2} \leq \omega \leq 10^{2} \mathrm{rad} / \mathrm{s}$, which result will be described later.

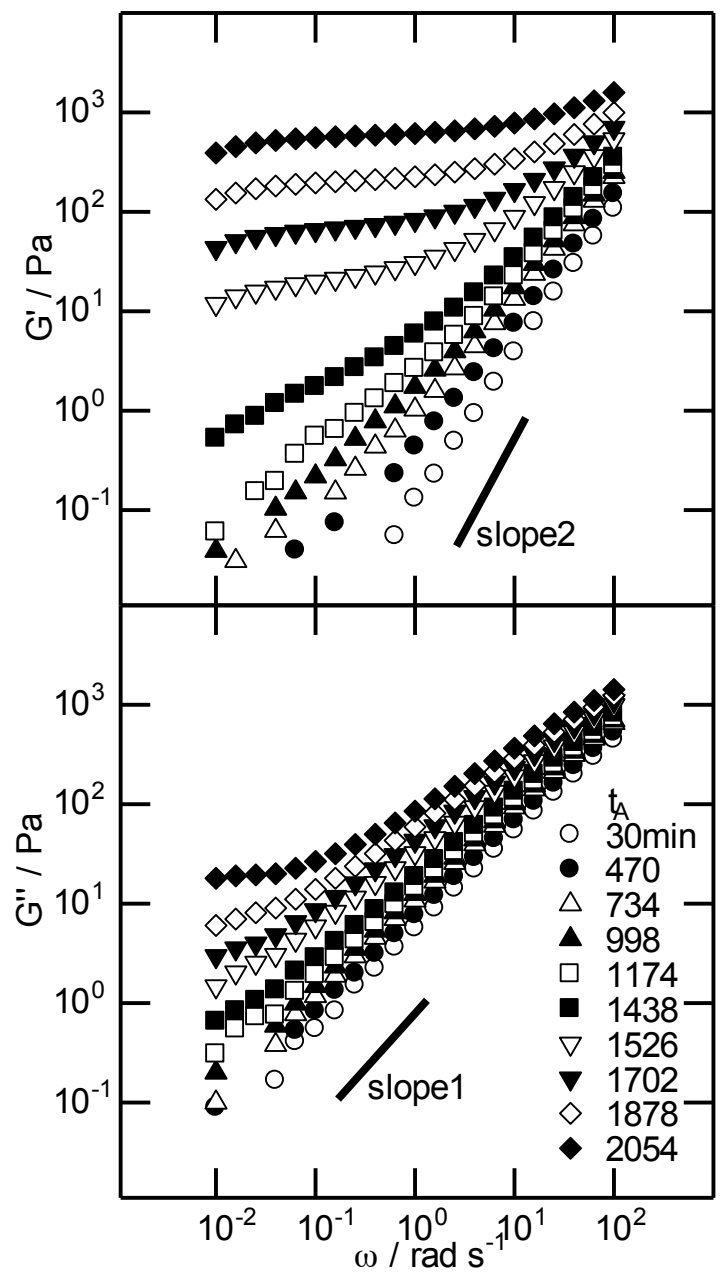

Figure 1. Storage modulus $G^{\prime}$ and loss modulus $G^{\prime \prime}$ of PAN-DMA solution of $14 \%, t_{\mathrm{F}}=17.5 \mathrm{~h}$, as a function of angular frequency $\omega$ for various ageing time $t_{\mathrm{A}}$ as indicated.

The sample solution prepared in this work is time-dependent system, where the evolution of network formation, and hence the evolution of viscoelasticity continuously takes place during the measurement. Therefore, in the strict meaning, it might not be stable to measure. However, as an approximate propensity, the power law relaxation in PAN solution is conceivable and can be discussed further.

The evolution of $G^{\prime}(\omega)$ and $G^{\prime \prime}(\omega)$ data with the progress of crosslinking can also be interpreted through the dynamic viscosity [11] given in eq.(2) using the extent of crosslink reaction, $p$, as a parameter.

$$
\eta^{*}(\omega, p)=\sqrt{G^{\prime 2}+G^{\prime \prime 2}} / \omega
$$

In the gelation of PAN-DMA solution, $p$ corresponds to the number of crosslinking loci, which is indexed by $t_{\mathrm{A}}$. For the results of viscoelastic measurements of the solution of $t_{\mathrm{F}}$ 
$=2 \mathrm{~h}$, the plot of $\eta^{*}$ vs $\omega$ was shown in Figure 2.

The solution of $t_{\mathrm{F}}=2 \mathrm{~h}$ is in a low viscosity, independent of frequency, and no elasticity as shown in the plot of $t_{\mathrm{A}}=30 \mathrm{~min}$. With the increase in $t_{\mathrm{A}}$, the viscosity increases and some elasticity sets in; the plot falling down to the right at high frequency expresses the elasticity. At low frequency, the viscosity is constant and equal to the steady-flow viscosity $\left(\eta_{0}\right)$; the plot has zero slope as expected from a viscoelastic liquid. The solution is in pre-gel region. Frequency dependent shear thinning is observed with an onset frequency which shifts to lower and lower values as it is closing in on the gel point. At $t_{\mathrm{A}}=2250 \mathrm{~min}, \eta^{*}$ follows a power law as written in eq.(3).

$$
\eta^{*}(\omega, p)=a S(i \omega)^{n-1}, a=\frac{\pi}{\Gamma(n) \sin (n \pi)}
$$

$S$ is a material constant, called as gel strength and $n$ is the relaxation exponent specific to the network structure. $i$ is imaginary unit. $t_{\mathrm{A}}=$ $2250 \mathrm{~min}$ is the gel point. It is also known as the critical gel. The onset of shear thinning has shifted to zero frequency, that is, the solution behaves as shear thinning at all frequencies. After passing the gel point, $\eta^{*}$ grows further with a great rise at low frequencies, where the slope of -1 is seen which is associated with the appearance of the plateau modulus of $g_{\text {inf }}\left(t_{\mathrm{A}}\right)$. The solution has gotten into the post gel region.

Four samples of $t_{\mathrm{F}}=2,8,14$ and $17.5 \mathrm{~h}$ were prepared and viscoelastic measurements were carried out in this study. The results of $n=0.86$, $0.87,0.87$ and 0.87 were obtained for the solutions of $t_{\mathrm{F}}=2,8,14$ and $17.5 \mathrm{~h}$, respectively. It can be said that the relaxation exponents are almost constant for the solutions of constant PAN concentration and various $t_{\mathrm{F}}$. In general, $n$ has a relationship with the extent of complexity of network at gel point $[12,13]$. Therefore, the length of freezing time might affect not on the network geometry but on the speed of network formation [1].

The power law behaviour is an expression of self-similar structure. To give analogies of 2-dimension mesh and the mesh pore size are of help to represent the structure. The mesh area following the self-similarity can be associated with the frequency window written below [14].

Three kinds of asymptote line can be given to Figure 2 as follows [15].

$$
\begin{aligned}
& \eta^{*}=\left\{\begin{array}{l}
\eta_{0}\left(t_{A}\right) \\
g_{\text {inf }}\left(t_{A}\right) / \omega
\end{array}\right. \\
& \eta^{*}=a S \omega^{n-1}
\end{aligned}
$$

Eqs.(4), (5) are for low frequency asymptote. Eq.(6) is for high frequency asymptote which is common to the pre-gel and the post-gel region. A characteristic frequency, $\omega^{*}$ can be defined by the intersect of $\eta_{0}$ with the power law relaxation of eq.(6) in the pre-gel region. $\omega^{*}$ divides between the gel behaviour and the liquid behaviour. $\omega^{*}$ value decreases as the gel point comes closer. The characteristic frequency, $\underline{\omega}^{*}$ can also be defined in the post gel region, given by the intersect of $g_{\text {inf }} / \omega$ with the power law of eq.(6). At frequencies above $\underline{\omega}^{*}$, the solution exhibits the behaviour of critical gel and at frequencies below $\underline{\omega}^{*}$ it behaves like a typical viscoelastic solid. $\underline{\omega}^{*}$ value increases with $t_{\mathrm{A}}$.

It follows that the frequency window to observe the self-similar structure is located in the higher frequency side and narrow in the initial stage of sol-gel transition. With the progress of the transition, the range expands to the lower frequency side. We have the widest range in the critical gel, and then the range shrinks again to the higher frequency side in the post-gel region.



Figure 2. Dynamic viscosity derived by eq.(2) for PAN-DMA solution of $t_{\mathrm{F}}=2 \mathrm{~h}$ as a function of angular frequency $\omega$ for various ageing time $t_{\mathrm{A}}$ as indicated. The plot of $t_{\mathrm{A}}=2250$ follows the relation $\eta^{*} \propto \omega^{n-1}$ as indicated by the solid line over all frequencies used in the measurement. The plot of $t_{\mathrm{A}}=3060$ follows the relation $\eta^{*} \propto \omega^{-1}$ as indicated by the dotted line, showing the plateau modulus.

In relation to the frequency window, there is the basic frequency-length relationship, in the sense that high frequency explores smaller length scales, and low frequency does large length scales. This is normally ruled by a dispersion relation (the Kramers - Kroning equation is one of the well-known examples) between the frequency of the experiment, $\omega$, relaxation time, $t$ and scale of observation, $L$, as shown in the relation of (7).

$$
\frac{1}{\omega} \sim t \sim L^{a}
$$

Low frequencies explore micro-scale structure in relation to the whole polymer or even the large parts of the material which is overwhelmingly dominant for flow properties, while very high frequencies are sensitive to relaxation within one chain or even to local jumps of side groups etc. 
However, no experimental works report the data of the actual gel sample for the relation of (7) This relation seems to be valid from the viewpoint of the mechanical spectroscopy. Therefore, to demonstrate the relation of (7) is an important issue.

The time constants $(\lambda)$ can be given in connection with the characteristic frequency.

$$
\begin{aligned}
& \lambda\left(t_{\mathrm{A}}\right)=\left(\frac{\eta_{0}\left(t_{A}\right)}{a S}\right)^{1 /(1-n)} \\
& \lambda\left(t_{\mathrm{A}}\right)=\left(\frac{a S}{g_{\text {inf }}\left(t_{A}\right)}\right)^{1 / n}
\end{aligned}
$$

Eqs.(8) and (9) are for pre-gel and post-gel regions, respectively. Data obtained from Figure 2 are summarised in Table 1.

The time constant of the crosslinking system varies significantly through sol-gel transition. In its earliest stage, the $\lambda$ value is very short, which corresponds to the motions of respective PAN chains. In the evolution of viscoelasticity, the most interesting part of $G^{\prime}$ and $G^{\prime \prime}$ data shifts to lower and lower frequencies with the progress of the transition. The shift to lower frequencies is expressed very strongly that the actual transition through the gel point cannot be observed in Figure 2 , because $\omega^{*}$ has shifted below $10^{-2} \mathrm{rad} / \mathrm{s}$ of the lower limiting frequency of the rheometer. Equivalently, the $\lambda$ value diverges to infinity and is not contained any longer in the relaxation spectrum. It is not until the solution reaches at post-gel region that the characteristic frequency becomes large again. Then the solid behaviour becomes measurable within the frequency range of the rheometer. $\lambda$ of the final network is again very short when the network has reached a high degree of crosslinking. The final value of $\lambda$ is expected to be of the same order as the initial one.

3.2 Power Law in Dynamic Mechanical Properties

Selfsimilarity seems to be the dominating feature of the critical gel of PAN-DMA solution. In this regards, an important proposition is shown that a material with self-similar structure has dynamic mechanical properties to follow a power law that $G^{\prime}(\omega)$ and $G^{\prime \prime}(\omega) \propto \omega^{n}$, and hence to follow eq.(3). Conversely, the material in which the power law is observable contains the structure of self- similarity. For PAN-DMA critical gel, it means the geometrically self-similar mesh.

It should be noted for the mechanical strength of the critical gel. It can be said that the critical gel flows. This is evidenced by the result that $G^{\prime \prime}$ values were higher than $G^{\prime}$ over all frequency range at $t_{\mathrm{A}}=998 \mathrm{~min}$ in Figure 1 . Although the network formed in the critical gel covers the finite sample size, in other words, the cluster has already percolated, it cannot sustain the stress applied by the rheometer cell. Furthermore, whether the network can sustain the stress or not, it will be a matter of time in which it is applied. Presumably, there might be some relationships among this time constant, the yield stress and $\lambda$, which are not elucidated at present.
Table 1. Asymptotic values derived from $\eta^{*}$ vs $\omega$ plot for PAN-DMA solution of $14 \%, t_{\mathrm{F}}=2 \mathrm{~h}$.

\begin{tabular}{ccccc}
\hline$n$ & $\begin{array}{c}t_{\mathrm{A}}-t_{\mathrm{A}-\mathrm{gel}} \\
(\mathrm{min})\end{array}$ & $\begin{array}{c}\eta_{0} \\
(\mathrm{~Pa} \cdot \mathrm{s})\end{array}$ & $\begin{array}{c}g_{\text {inf }} \\
(\mathrm{Pa})\end{array}$ & $\begin{array}{c}\lambda \\
(\mathrm{s})\end{array}$ \\
\hline 0.86 & 1680 & 14.73 & - & 0.15 \\
& 1050 & 16.36 & - & 1.79 \\
& 600 & 20.56 & - & 7.87 \\
& 0 & - & - & - \\
& 540 & - & 2.97 & 56.58 \\
& 810 & - & 8.49 & 15.78 \\
& 1080 & - & 23.18 & 1.59 \\
\hline
\end{tabular}

The data that $G^{\prime}(\omega)$ tends to fall at the terminal region in $t_{\mathrm{A}}=2054 \mathrm{~min}$ in Figure 1 also reflect the result that the network formed could not sustain the stress application of long time; the relaxation of next lower frequency range is imminent.

To return to the discussion on power law and the network structure, the relation between $\omega^{*}$ and a correlation length $\xi$ is explained in terms of the mesh geometry. In the pre-gel state, PAN molecules are crosslinked into clusters which are weak solids of small spatial dimension. The finite cluster itself is a piece of macromolecule, which can be defined as a polymeric fractal[7], with a distribution of "defects" studied in the percolation problem[16], such as loose ends, loops, variation of the multiplicity in the crosslinking loci etc. In this sense, fractal is not the geometrical idea but the actual object. Assuming there are large clusters in the solution containing smaller clusters and uncrosslinked PAN chains, the viscoelasticity measurement is mostly sensitive to the size of a typical cluster. So, $\xi$ is the size of a typical cluster in the pre-gel region where only finite clusters are contained.

Afterwards, $\xi$ becomes larger and larger until it extends across the entire sample. The size of the typical cluster becomes infinite at $t_{\mathrm{A}-\mathrm{gel}}$. The self-similar structure can be thought of as the mesh geometry. The parallel data seen in $G^{\prime}$ and $G^{\prime \prime}$ over $\omega$ of three decades indicates that there is no dominant length scale in the mesh geometry. That is, the correlation length is infinite and on each scale of observation a similar structure can be found.

The post gel state shows $g_{\text {inf }}$, for the first time, indicating a certain amount of elastic active chain. The mesh is no longer self-similar at the whole size, where $\xi$ can be given by the self-similar region in the mesh. With the progress of the gelation, the self-similar regions decrease, and hence $\xi$ decreases. The power law at larger frequencies appears again with measuring $\xi$, whereas the solid behaviour is present at lower frequency. The fractal information of the infinite cluster disappears as more and more chains are linked. The final state, if most of the active sites have reacted, is a rubber which is formed by a 
very large number of crosslinking loci and has a finite modulus of $g_{\text {inf }}$ for low frequency. In the sense of $G^{\prime}$ and $G^{\prime \prime}$ data and the mesh geometry, the short distance behaviour has less relaxation, which is probed only at high frequency far above the frequency window of the experiment, but the large scale elasticity of a size larger than a single sub-chain, i.e., a chain between two crosslinking loci or a dangling chain, is meaningful.

Taking account of eq.(7), the relation between $\omega^{*}$ and $\xi$ can be expressed as follows.

$$
\omega^{*} \sim \xi^{-\alpha}
$$

Several relationships are presented in this work among length scale, time constant, frequency and dynamic viscosity. These suggest a close analogy with the percolation problem. The examination on the percolation idea in detail is expected for the scope of further study, where the rigid bonds are located on the Bethe lattice or a square lattice. The main difference from the application of the usual percolation problem is that the bonds must be replaced by flexible polymer chains having the fractal dimension.

\section{CONCLUDING REMARKS}

For the sol-gel transition of PAN-DMA solution prepared with the freezing and thawing method, the parallel data, and hence the power law relaxation, are observed in $G^{\prime}(\omega)$ and $G^{\prime \prime}(\omega)$ over frequencies of three decades. The power law data as expressed by the dynamic viscosity can be interpreted in terms of geometrical properties of the network formation, which was analogously substituted with 2-dimension mesh in this work. Several relationships are presented among length scale, time constant, frequency and dynamic viscosity to follow the geometrical variation with the progress of the transition.

\section{REFERENCES}

[1] M. Yasuhara, Y. Tanaka, S. Saeki, Trans. Mat. Res. Soc. Jpn., 26(2), 593-596(2001)

[2] M. Bercea, S. Morariu, C.-E. Brunchi, International J. Thermophysics, 30(4) 1411-1422(2009)

[3] T.A.Vilgis, H.H.Winter, Colloid Polym. Science, 266, 494-500(1988)

[4] H. H. Winter, Progr. Colloid Polym. Science, 75, 104-110(1987)

[5] J.Beckmann, D.Zenke, Colloid Polym. Sci., 271, 436-445(1993)

[6] R. Chiang, J. Polym. Sci. Part A: General Papers, 3(5), 2019-2026(1965)

[7] M. Muthukmar, J. Chem. Phys., 83(6), 3161-3168(1985)

[8] M. E. Cates, J. Physique, 46, 1059-1077(1985)

[9] T. Shibukawa, M. Sone, A. Uchida, K. Iwahori, J. Polym. Sci. Part A-1 : Polym. Chem., 6(1), 147-159(1968)

[10] J. A. Riddick, W. B. Bunger, T. K. Sakano, Organic Solvents Physical Properties and Methods of Purification, 4th Ed., pg.661(1986) John Wiley and Sons, NY.

[11] W. W. Graessley, Adv. Polym. Sci., 16, 163(1974)

[12] J. C. Scanlan, H. H. Winter, Macromolecules, 24, 47-54(1991)

[13] Anna-Lena Kjøniksen, Bo Nyström, Macromolecules, 29, 5215-5222(1996)

[14] X. Jiao, Y. Tanaka, Polym. Preprints, Jpn., $60,874(2011)$

[15] Y. Tanaka(2012) Viscoelastic Properties for Sol-Gel Transition, Rheology, J. D. Vicente(Ed.), ISBN: 978-953-51-0187-1, InTech, Available from: http://www.intechopen.com/books/rheology /viscoelastic-behaviour-for-gelation

[16] D. Stauffer, A. Coniglio, M. Adam, Adv. Polym. Sci., 44, 103-158(1982)

(Received October 26, 2012; Accepted December 12, 2012) 